\title{
Underwater Mass Spectrometers for in situ Chemical Analysis of the Hydrosphere
}

\author{
R. T. Short, D. P. Fries, M. L. Kerr and C. E. Lembke \\ Center for Ocean Technology, College of Marine Science, University of South Florida, St. Petersburg, Florida, \\ USA \\ S. K. Toler, P. G. Wenner and R. H. Byrne \\ College of Marine Science, University of South Florida, St. Petersburg, Florida, USA
}

\begin{abstract}
Underwater mass spectrometry systems can be used for direct in situ detection of volatile organic compounds and dissolved gases in oceans, lakes, rivers and waste-water streams. In this work we describe the design and operation of (1) a linear quadrupole mass filter and (2) a quadrupole ion trap mass spectrometer interfaced, in each case, with a membrane introduction/fluid control system and packaged for underwater operation. These mass spectrometry systems can operate autonomously, or under user control via a wireless rf link. Detection limits for each system were determined in the laboratory using pure solutions. The quadrupole mass filter system provides detection limits in the 1-5 ppb range with an upper mass limit of $100 \mathrm{amu}$. Its power requirement is approximately 95 Watts. The ion trap system has detection limits well below 1 ppb, an upper mass limit of $650 \mathrm{amu}$ and MS/MS capability. Its power consumption is on the order of 150 Watts. The present membrane limits analysis to non-polar compounds (<300 amu) with analysis cycles of 5-15 minutes. Deployments of both types of instruments are described, along with a discussion of the challenges associated with in-water mass spectrometry and descriptions of alternative in-water mass spectrometer configurations. (J Am Soc Mass Spectrom 2001, 12, 676-682 ) (C) 2001 American Society for Mass Spectrometry
\end{abstract}

S tandard methods for analysis of aqueous systems typically involve collection of samples and delivery to a laboratory for analysis [1]. Inherent in this practice is the possibility of both contamination and loss of analytes, particularly in the case of highly reactive or volatile species. In addition, this type of sampling severely limits both spatial and temporal sampling densities. A particularly important limitation of long collection/analysis cycles is the inability to implement adaptive sampling. Appropriate monitoring of dynamic biogeochemical systems requires rapid sampling adaptations in response to rapidly varying analyte distributions [2]. Intelligent sampling strategies are required to adequately characterize vast bodies of water that influence, and are influenced by, human activities. Since mass spectrometry is arguably the most versatile of chemical sensors, we have undertaken development of in situ mass spectrometry systems capable of real-time, adaptive in-water analyses.

Although mass spectrometry has been used in the

Published online May 1, 2001

Address reprint requests to R. T. Short, Center for Technology/College of Marine Science, 140 Seventh Avenue South, University of South Florida, St. Petersburg, FL 33701-5016. E-mail: tshort@marine.usf.edu laboratory for an extremely wide variety of chemical analyses, from precise isotopic ratio measurements [3] to DNA sequencing [4-6], no single configuration of mass analyzer and sample interface is appropriate for all types of measurements. This is evidenced in recent reviews of field analytical techniques [7] and advances in miniaturization of mass spectrometers [8]. Accordingly, we have chosen a modular approach for development of immersion mass spectrometers. Initially, simpler designs are used to integrate available components, and simultaneously new subsystems are being developed for evolving reconfigurations capable of accessing a wider range of chemical species.

The primary challenges faced in creating underwater mass spectrometry (MS) systems are related to the necessity of performing mass spectrometry in a vacuum. Analytes must be transported from the aqueous environment into a vacuum system, underwater. Since analysis of aqueous samples inevitably increases gas loads on vacuum pumps, use of entrainment or capture pumps would entail frequent regeneration. Alternatively, if throughput pumps are used in a closed system, the inevitable increase in exhaust pressure of these pumps will eventually degrade pump operation. Since ambient underwater pressure increases by approxi- 
mately 1 atmosphere with 10 meter depth increments, regeneration of entrainment pumps or decompression of pump housings becomes impractical at substantial depths.

Our prototype system deployments have initially involved shallow water proof-of-concept operations at depths less than 30 meters. Future modifications are planned that will allow deployment at increased depth. Our primary initial focus has been analysis of the coastal ocean for natural and anthropogenic organics. Membrane introduction was chosen as the initial sample acquisition technique because of its simplicity and because the technique has very low detection limits for dissolved gases and volatile organic compounds [9-11]. The membrane interface works equally well with both fresh water and seawater samples [10]. Our vacuum system design [12, 13] allows continuous underwater operation for up to 12 days. Strategies for longer-term underwater vacuum maintenance are presently being investigated, but these will not be addressed herein. We note here that researchers at the Massachusetts Institute of Technology are independently pursuing development of an underwater membrane introduction mass spectrometer [14].

\section{Experimental Apparatus}

\section{Underwater Membrane Introduction/Quadrupole Mass Filter System}

The mass analyzer chosen for our first underwater mass spectrometer system was a linear quadrupole mass filter (Transpector 2 Residual Gas Analyzer, Inficon Inc., Syracuse, NY) coupled to a membrane introduction interface (MIMS Technology, Inc., Palm Bay, FL). The membrane introduction probe employs a polydimethylsiloxane (PDMS) capillary (approximately $15 \mathrm{~mm}$ length, $0.64 \mathrm{~mm}$ inner diameter, and $1.19 \mathrm{~mm}$ outer diameter) that is connected to two stainless steel tubes in a U-shaped geometry. The capillary exterior, located very close to the mass filter ion source, is exposed to the vacuum of the mass spectrometer. Water entering the capillary is heated in-line to $60{ }^{\circ} \mathrm{C}$ using an Omega model CN491A-D1 temperature controller (Omega, Stamford, CT). This module controls the current in two embedded heater cartridges with feedback from a temperature sensor in the membrane probe assembly's heater block.

A multi-channel peristaltic pump (Pump Express/ ALITEA AB, Chicago, IL) and a 2-position rotary switching valve (Valco Instruments, Co., Houston, TX) comprise the fluid control system that is used to alternately direct deionized water and sample water to the membrane interface. Narrow-bore PEEK tubing (Upchurch Scientific, Inc. Oak Harbor WA) is used for component interconnections of the fluidic system. The peristaltic pump is used to direct both deionized water and sample water through the system at a nominal rate of 0.5 to $1.0 \mathrm{ml} / \mathrm{min}$. Two types of fluidic-control systems have been employed: flow injection and fluid stream switching. These systems involve the use of two different types of dual-position multi-port switching valves. Both systems allow comparison of sample analyte intensities and background intensities by alternately introducing sample and deionized water to the membrane. Our initial fluid system used flow injection to introduce a reproducible volume of sample into the analyzer. The system utilized a 6-port rotary switching valve that contained a $1 \mathrm{ml}$ sample loop. The loop was continuously filled off-line, with periodic switching in-line to allow the loop contents to pass through the membrane capillary. Our current configuration uses a valve that does not have a sample loop, allowing a more flexible fluid-stream switching method to introduce samples. This dual-position 4-port valve allows sample water and deionized water to be alternately directed to the membrane interface. In this manner, the volume of the introduced sample is determined by the pumping speed and the time that the valve remains in position. The advantage of this procedure is that the volume of sample introduced to the analyzer can be varied over a continuous range and optimized for each analysis (without change of hardware). Further discussion of this variable sample volume technique is found below.

A vacuum housing for the quadrupole mass filter was designed to ensure that compounds entering the vacuum initially pass through the ion source. Vacuum is maintained by a V70LP MacroTorr pump (Varian Vacuum Products, Lexington, MA) backed by two Model N84.0-11.98 diaphragm pumps (KNF Neuberger, Inc., Trenton, NJ) connected in series. The turbo/drag pump and brushless-motor diaphragm pumps are both powered by 24 VDC and consume on the order of $45 \mathrm{~W}$ during operation. The $\sim 1$ torr backing pressure required by the V70LP pump is provided by the diaphragm pumps and, with the membrane-introduction interface connected and water flowing through the membrane capillary, the pressure in the mass spectrometer is $\leq 10^{-5}$ torr.

The Transpector 2 residual gas analyzer (RGA) provides two modes of operation. Either full mass scans or intensities of selected ion masses can be obtained in the 1-100 amu range. The latter mode (selected ion monitoring) has been used in our flow-injection analyses. The RGA is powered by 24 VDC, consumes approximately $24 \mathrm{~W}$, and communicates via an RS-232 port with a $166 \mathrm{MHz}$ CardPC (Cell Computing, San Jose, CA) containing a $144 \mathrm{MB}$ DiskonChip. The embedded computer is powered by 5 VDC and consumes a maximum of $5.3 \mathrm{~W}$ during routine operation. Communication and control of the embedded computer can be provided by an external laptop computer through an Ethernet port. This can be accomplished using either a hardwire connection or a wireless rf link.

For operation of the Transpector RGA in the selected ion monitoring mode we have chosen a dwell time of $512 \mathrm{~ms}$ at each monitored ion mass. Including settling time and mass spectrometer overhead operations, the analyzer can cycle through 18 selected masses in less than 10 s. A background intensity, determined from 


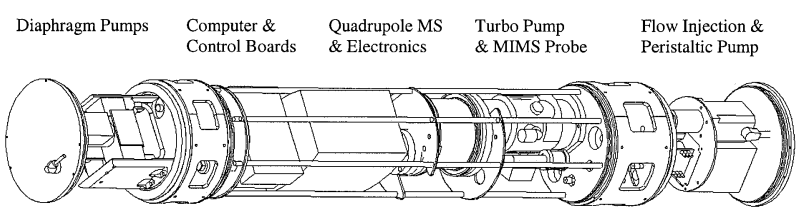

Figure 1. Schematic of the three-pressure-vessel system for the underwater membrane-introduction quadrupole mass filter system.

user-selected regions where no ions are expected, is subtracted from target ion intensities. The Transpector employs either a Faraday cup detector or an electron multiplier detector in the form of a single microchannel plate. For typical trace species analysis the electron multiplier detector is operated at $1050 \mathrm{~V}$.

For underwater deployment (Figure 1) the membrane introduction/quadrupole mass filter system is packaged in a series of three pressure vessels. The modular threepressure vessel approach was chosen for several reasons. The fluid-control components, housed in a separate vessel, represent a stand-alone system that can be incorporated in other types of analytical systems. In addition, this approach isolates multiple fluid connections from sensitive electronic components in the primary mass spectrometer housing, thereby minimizing potential damage from small water leaks. The system's diaphragm pumps are also housed in a pressure vessel separate from the mass analyzer system. Thermal coupling of the pumps to the pressure vessel endcap effectively dissipates heat generated during operation. Most importantly, a dedicated pressure chamber extends the endurance of the underwater vacuum system for time series deployments. Diaphragm pumps, the roughing-pump stage of MS vacuum system, exhaust directly into their pressure housing. Although the gas throughput from the high-vacuum region is minimal after the vacuum housing is pumped down, the pressure inside the closed roughing-pump system will eventually exceed an effective operational level. By housing the diaphragm pumps in their own separate pressure vessel, and evacuating this container to approximately one quarter of atmospheric pressure, we have demonstrated that the vacuum system can be operated in a closed underwater environment for up to 12 days.

The third system housing, containing the mass analyzer system, the turbo pump and controller, the embedded computer and other electronics, is located between the sample introduction and vacuum modules as shown in Figure 1. Internal components are thermally coupled to the walls of the pressure vessel (similar to the diaphragm pumps) and a small fan is used to circulate the air inside the housing. Use of the underwater environment as a heat sink has proven effective even in relatively warm Florida waters. The three 0.19-m-diameter cylindrical aluminum pressure vessels for this system are anodized for extended underwater life. The housings all have o-ring seals and waterproof electrical interconnections. Fluidic components are interconnected via a polyetheretherketone (PEEK) tubing, and a stainless steel vac-

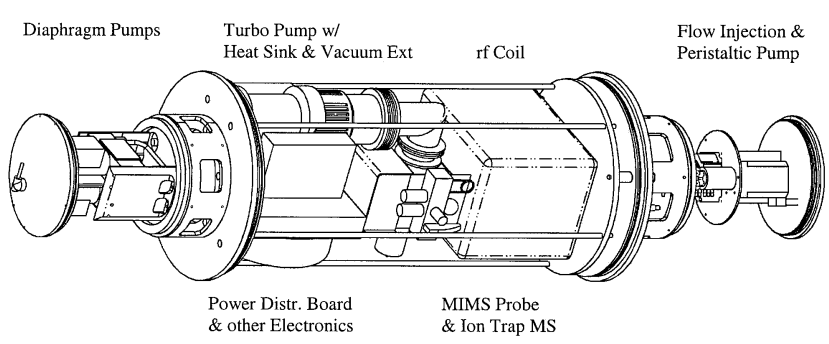

Figure 2. Schematic of the three-pressure-vessel system for the underwater membrane-introduction ion trap mass spectrometer system. The first and third vessels are identical to those used on the quadrupole mass filter system.

uum tube couples the mass spectrometer and diaphragm pump housings. The system consumes $95 \mathrm{~W}$ during routine operation and, although designed for deployment on state-of-the-art autonomous underwater vehicles, can also be deployed as a moored sensor. The pressure vessel system is $1.40 \mathrm{~m}$ in length, weighs $39 \mathrm{~kg}$ in air, and is nearly neutrally buoyant in water. The operational depth of the present design is limited by the fluid system components to $70 \mathrm{~m}$ or less in either saltwater or fresh water.

\section{Underwater Membrane Introduction/Ion Trap Mass Spectrometer System}

Development of an underwater membrane-introduction ion trap MS system was undertaken in anticipation of its superior analytical capabilities relative to the quadrupole mass filter. These improvements include a wider mass range (up to $650 \mathrm{amu}$ ), a factor of twenty increase in sensitivity [12] even while acquiring full mass spectra during each cycle (as opposed to linear quadrupole selected-mass monitoring), and the possibility of performing multiple-stage mass spectrometry (MS/MS [15] and $\mathrm{MS}^{\mathrm{n}}$ [16]). Packaging the ion trap for underwater use, however, required substantial redesign in comparison to the quadrupole mass filter (discussed below).

Figure 2 shows our modified Saturn 2000 Ion Trap Mass Spectrometer (Varian Analytical, Walnut Creek, CA) combined with a membrane interface (functionally identical to that used on the underwater quadrupole system) and packaged for underwater use. The membrane interface is attached to the ion trap vacuum housing at the location normally occupied by a gas chromatograph (GC) transfer line. In this manner analytes that diffuse out of the membrane are forced to enter the ion trap, where they are subsequently ionized. Helium buffer gas is not used in this arrangement since neutral water vapor and dinitrogen serve as sufficient collision gases for the non-polar species introduced through the membrane. The modular mass spectrometer system design facilitates use of the quadrupole fluidic and diaphragm pump modules with the ion trap MS pressure vessel. However, as seen in Figure 2, the ion trap pressure vessel is slightly larger than that of the quadrupole mass filter system. In order to fit the Saturn 
2000 ion trap MS inside the $0.31 \mathrm{~m}$ diameter of the pressure vessel, several modifications of the system were required. A redesigned and miniaturized power distribution board was configured for 24 VDC operation. The waveform generation board (SAPWAVE) was redesigned as two smaller boards. The V70 turbomolecular pump was replaced with a V70LP turbo/drag pump and the vacuum system was reconfigured to fit within the $0.31 \mathrm{~m}$ dia tube. The embedded computer for data acquisition is a Cell Computing (San Jose, CA) modular Plug-N-Run PII 333MHz PC System with an IBM 340MB Microdrive. The embedded PC communicates with the Saturn 2000 system via a National Instruments PCMCIA GPIB (Personal Computer Memory Card International Association, General Purpose Interface Bus) interface card. The entire system, including flow injection components and diaphragm pumps, weighs approximately $68 \mathrm{~kg}$ in air, is nearly neutrally buoyant in water and is $1.35 \mathrm{~m}$ in length. During operation it consumes on the order of $150 \mathrm{~W}$.

Typical operational parameters of the reconfigured Saturn 2000 Ion Trap MS for membrane interface flow injection analyses are as follows: Electron emission current is from 5-30 $\mu \mathrm{A}$ depending on the application. Ionization is performed with a 35-amu low-mass cutoff to exclude the water vapor and nitrogen ions that are introduced through the membrane. Average mass scans from 40-250 amu are plotted every $5 \mathrm{~s}$ during analyses. We limit the mass spectral acquisitions to this range because the transmission characteristics of the membrane set a practical upper analysis limit of around $300 \mathrm{amu}$. Automatic gain control (AGC) is used with an rf ramp to eject ions up to $650 \mathrm{amu}$ before each ionization period and mass scan. The target ion count for AGC is set well above the anticipated ion count to ensure that the entire $60 \mathrm{~ms}$ ionization time is used for trace analysis. Water is heated to $35^{\circ} \mathrm{C}$ in the membrane introduction probe, a lower value than used for the quadrupole system. The ion trap manifold heater is set to $50{ }^{\circ} \mathrm{C}$ while the trap heater is held at $80{ }^{\circ} \mathrm{C}$. These temperatures provided an optimized signal-to-noise ratio for trace VOC analysis in water.

\section{Results and Discussion}

\section{Underwater Membrane Introduction/Quadrupole Mass Filter System}

Performance of the membrane-introduction quadrupole MS assembly was evaluated in the laboratory using deionized water solutions of volatile organic compounds (VOCs) at known concentrations. Previous measurements comparing analyses of deionized water and seawater [10] produced no membrane introduction matrix effects. Figure 3 shows results from flow injection analyses of solutions containing toluene and dimethylsulfide (DMS). One ml samples with analyte concentrations of $1 \mathrm{ppb}, 5 \mathrm{ppb}, 10 \mathrm{ppb}$ and $20 \mathrm{ppb}$ were analyzed. Data for the major diagnostic ions $(\mathrm{m} / \mathrm{z} 91 \mathrm{for}$ toluene and $\mathrm{m} / \mathrm{z} 62$ for DMS) demonstrate that both

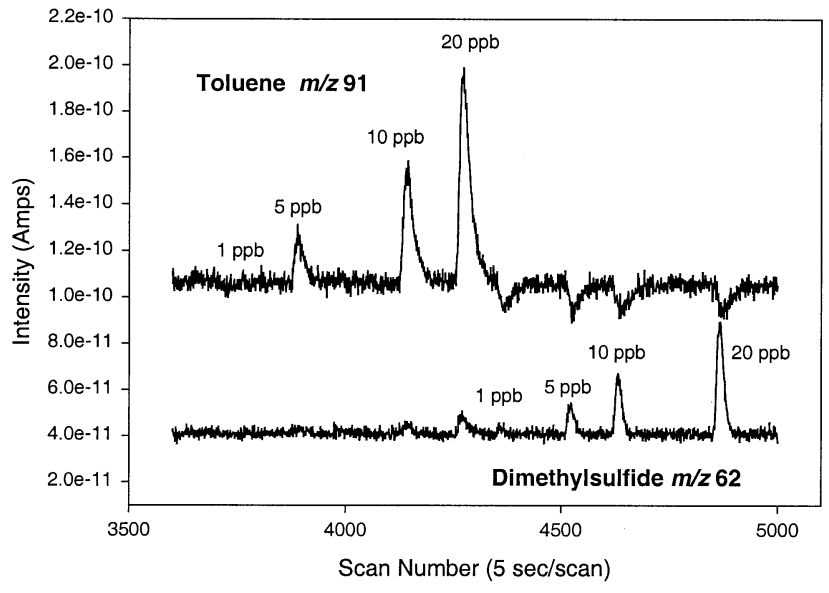

Figure 3. Laboratory data from analysis of standards using the underwater quadrupole MS system. Concentrations noted in the diagnostic ion traces correspond to flow-injection analyses of $1 \mathrm{ml}$ solutions of toluene and dimethylsulfide.

compounds were clearly detectable at 5 ppb. Furthermore, at $1 \mathrm{ppb}$ DMS was detectable with a signal-tonoise ratio of approximately 2:1.

Background intensities, typically attributed to the residual gas in the vacuum system, were higher for $\mathrm{m} / \mathrm{z}$ 91 than for $m / z$ 62. In this case, we also had background contributions from the deionized "blank" water. Contamination of the deionized water was evidenced by a decrease in $\mathrm{m} / \mathrm{z} 91$ intensity (in the last four injections) during analysis of DMS solutions, which were made using uncontaminated water. We later confirmed that this contamination came from the medical-grade silicone flexible bag used to contain the deionized water. We currently use Tedlar bags (Cole Palmer, Vernon Hills, IL) which do not introduce contaminants into the deionized water. Use of uncontaminated deionized water lowered our toluene detection limit to approximately 1 $\mathrm{ppb}$. A minor $\mathrm{m} / \mathrm{z} 62$ fragment of toluene was also detectable in analyses of the higher concentration (10 and 20 $\mathrm{ppb}$ ) standards. These results accentuate the limitation of single-stage mass spectrometry (particularly, selected ion monitoring) for analysis of complex samples.

The first underwater tests of the system were performed in a large water tank at the University of South Florida Center for Ocean Technology (COT). The tank was filled with approximately 30,000 1 of municipal water. The quadrupole MS system was suspended in the tank at a depth of approximately $0.5 \mathrm{~m}$. Waterproof cables were connected to provide 24 VDC system power and real-time monitoring of data. Operation of the flow-injection valve was accomplished using a wireless keyboard and mouse near the side of the water tank. Figure 4 displays in situ data obtained for two monitored ion masses, $\mathrm{m} / \mathrm{z} 83$ and $\mathrm{m} / \mathrm{z}$ 91. The peaks in the data correspond to repetitive flow injection analyses of tank water. Each data point (scan number) represents a $16 \mathrm{~s}$ cycle interval. Total analysis cycles were approximately 15 minutes to allow ion traces to return to back- 


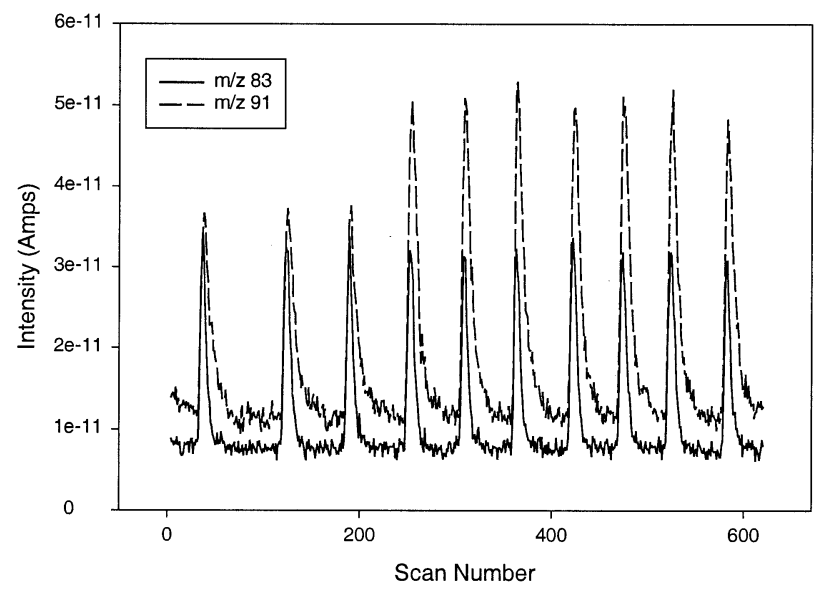

Figure 4. In situ data from the quadrupole MS system immersed in a large tank of municipal water. The $m / z 83$ ion is diagnostic of chloroform and the $\mathrm{m} / \mathrm{z} 91$ ion is diagnostic of toluene. The increase in $\mathrm{m} / \mathrm{z} 91$ during the fourth flow-injection analysis corresponds to $3 \mathrm{ml}$ of toluene added to the 30,000 l of tank water. Each scan represents a $16 \mathrm{~s}$ analysis cycle.

ground level. The $\mathrm{m} / \mathrm{z} 83$ peaks correspond to chloroform, which is routinely found in St. Petersburg, FL domestic water. This represents a concentration on the order of 50 ppb (determined by comparison with standards having known concentrations). Minor diagnostic ions of chloroform are not shown, but were also present in these analyses. The $\mathrm{m} / \mathrm{z} 91$ data corresponds to toluene, which is not normally found at this level in domestic water. We attribute the initial presence of toluene in injected samples to contamination from previous activities in the water tank, and outgassing of the tank walls.

To demonstrate the sensitivity and response time of the in situ MS, a 3\% toluene/methanol solution (3 $\mathrm{ml}$ of toluene in $97 \mathrm{ml}$ methanol) was added to the tank water approximately 5 meters from the inlet of the mass spectrometer. The addition occurred between the third and fourth sampling cycles in the series. The water was then turbulently stirred to speed mixing throughout the tank. The increase in intensity at $\mathrm{m} / \mathrm{z} 91$ (beginning with the fourth peak in the series, several minutes after the addition of toluene) indicated a concentration increment equal to approximately $50 \mathrm{ppb}$ according to previous measurements using standards of known concentration. If the toluene had been evenly dispersed throughout the 30,000 1 tank, the expected concentration change would be $100 \mathrm{ppb}$. The variation in $\mathrm{m} / \mathrm{z} 91$ peak intensity after dispersion of the toluene is larger than the typical statistical variation of the system and is attributed to small local concentration variations from turbulent mixing of the tank water. These measurements demonstrated the operational viability of the underwater mass spectrometry system and confirmed the system's sensitivity to small VOC concentration variations.

In order to demonstrate autonomous operation, the underwater quadrupole MS system was installed on the Florida Atlantic University (FAU) Ocean Explorer (OEX) autonomous underwater vehicle (AUV) [17, 18].

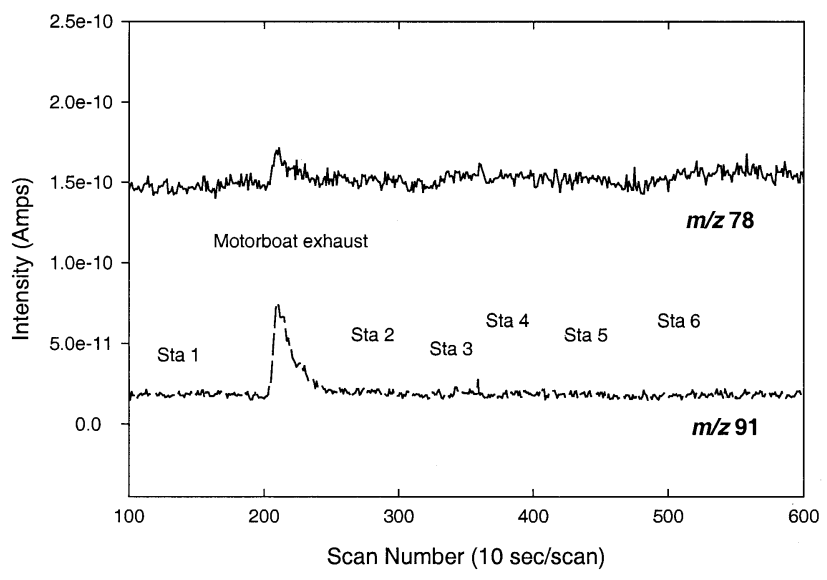

Figure 5. Field data from towed underwater deployment of the quadrupole MS system in Bayboro Harbor. The $\mathrm{m} / \mathrm{z} 78$ ion is diagnostic of benzene and the $\mathrm{m} / \mathrm{z} 91$ ion is diagnostic of toluene. Sta \#s represent locations where Harbor water was analyzed. The single peak in each ion trace corresponds to analysis of water contaminated with outboard motor exhaust.

The MS system was powered using two lead-acid battery packs (240 Watt-hours each) that allow up to 5 hours of continuous system operation. A valve-control software program was created to cycle the flow injection valve and automatically inject samples during AUV operation. This software operates using the embedded PC in parallel with the Transpector data acquisition software. A cycle period of 12 minutes was chosen for compatibility with typical flow-injection peak widths, which are primarily determined from diffusion rates through the membrane interface.

In collaboration with FAU personnel we successfully deployed the mass spectrometer/AUV assembly on three separate routes over the course of two days. The AUV deployment and retrieval platform was the R/V Subchaser $(10.7 \mathrm{~m})$. The first AUV deployment, in Bayboro Harbor (adjacent to the USF College of Marine Science, St. Petersburg, FL), lasted for approximately one hour. The second of two subsequent deployments (Tampa Bay) lasted for more than 3 hours. The data from each of these runs, however, showed no substantial evidence of VOC contamination above the ppb detection limits of the system. Nonetheless, these tests demonstrated, over periods of several hours, the first autonomous operation of an AUV-deployed mass spectrometer.

The underwater mass spectrometry system was also towed behind a small boat in Bayboro Harbor for collection of in situ MS data. The boat was propelled by a battery-powered trolling motor to avoid gasolineexhaust contamination of the water being sampled by the underwater MS. Our towed operations allowed sampling in areas that are inaccessible to present generation AUVs (e.g., narrow creeks and crowded marinas). Flow-injection data were acquired by allowing the underwater MS to analyze samples at a number of specific locations. In situ results for two selected masses, $m / z 78$ and $m / z$ 91, are shown in Figure 5. 


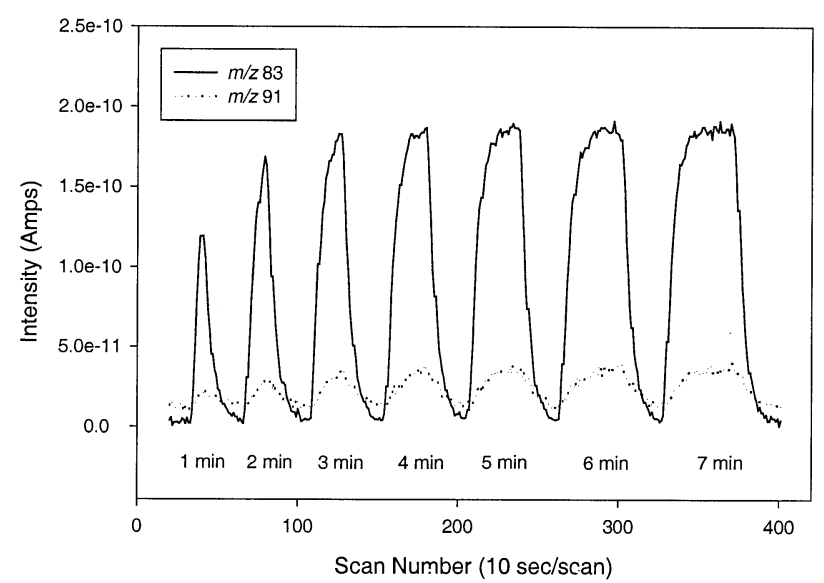

Figure 6. In situ data obtained using the quadrupole MS system demonstrates variable-volume sampling. The sample volume analyzed is determined by pumping speed $(1 \mathrm{ml} / \mathrm{min})$ and dwell time in the sampling position (noted in figure for each peak). Deionized water is analyzed between samples.

Sample locations are denoted as "Sta \#" in the figure. There was no significant increase above background for these compounds (nor any other monitored masses) at any of the locations. The only detectable increase was observed when a gasoline powered outboard motor was running nearby. This is shown by the increase in both traces between Sta 1 and Sta 2 . The $\mathrm{m} / \mathrm{z} 78$ and $\mathrm{m} / \mathrm{z}$ 91 ions are diagnostic of benzene and toluene, respectively, which are VOC components of typical gasoline mixtures. We also collected water samples concurrently at each location for subsequent laboratory analysis using a membrane-introduction ion trap mass spectrometer, with lower detection limits than the quadrupole system (see discussion below).

All of the analyses presented above were obtained using a flow-injection valve with a fixed-volume $1 \mathrm{ml}$ sample loop. We have since replaced this valve with one that contains no sample loop, and thus use a fluid-stream switching approach to introduce samples. The volume of sample analyzed using the new valve is solely determined by the water pumping speed and the time the valve remains in position for sample analysis. Accordingly, the volume of deionized water analyzed in the second valve position is also dependent only on pumping speed and time. This new valve thus allows the sample volume to be continuously varied and adapted to analyte acquisition conditions. This capability is illustrated in Figure 6, which shows $\mathrm{m} / \mathrm{z} 83$ and $\mathrm{m} / \mathrm{z}$ 91 ion traces for in situ analyses of munincipal water in the COT water tank. With a pumping speed of 1 $\mathrm{ml} / \mathrm{min}$, as used for these analyses, the sample volume was varied in $1 \mathrm{ml}$ steps from 1 to $7 \mathrm{ml}$ by changing sample position dwell time. From these measurements it is clear that a $1 \mathrm{ml}$ sample ( 1 min dwell time) does not provide optimum signal-to-noise ratios for quantification of these compounds. Maximum peak height is not reached until a $3 \mathrm{ml}$ sample ( $3 \mathrm{~min}$ dwell time) is introduced. A steady-state peak intensity is seen for

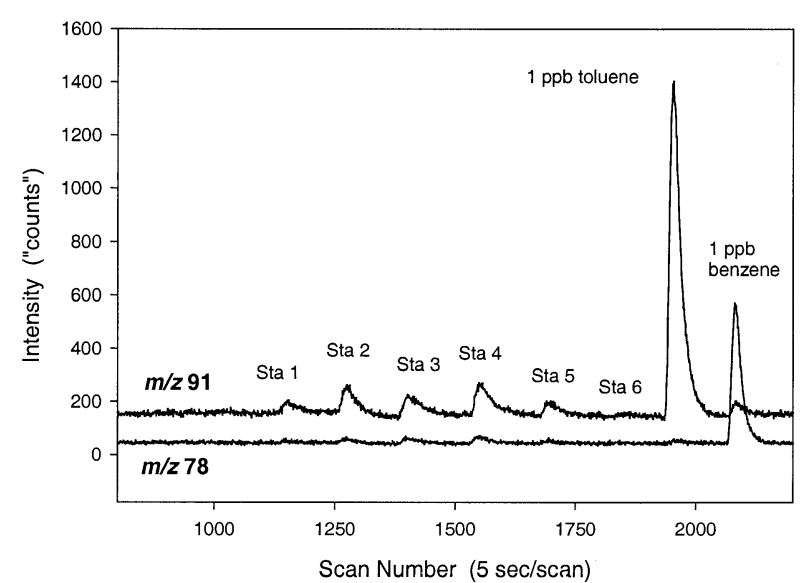

Figure 7. Laboratory data from ion trap MS analysis of water samples that were obtained during towed-deployment of the quadrupole MS system. The $\mathrm{m} / \mathrm{z} 78$ ion is diagnostic of benzene and the $\mathrm{m} / \mathrm{z} 91$ ion is diagnostic of toluene. Analyses of samples are compared with $1 \mathrm{ppb}$ standards.

analyses of samples greater than $3 \mathrm{ml}$. This capability allows rapid field adaptation of sampling strategies in response to observations. The system also facilitates fundamental studies of membrane transmission properties.

\section{Underwater Membrane Introduction/Ion Trap Mass Spectrometer System}

Compelling motivation for development of an underwater ion trap MS system arose from predicted performance gains and laboratory measurements that routinely exhibited detection limits 20 times better than those of the quadrupole system. The ion trap also offers additional advantages relative to the quadrupole system, such as full mass scans for each analysis, extended mass range and MS/MS capability. It should be noted however, that the space-charge limitations of the ion trap MS require that ionized water and ionized $\mathrm{N}_{2}$ be excluded from the trap. This was accomplished by using a low-mass cutoff (35 amu) during ionization periods. This operational necessity makes the ion trap system inappropriate for analysis of low molecular weight compounds (below ca. $40 \mathrm{amu}$ ). In contrast, the quadrupole membrane introduction mass spectrometry (MIMS) system does not have such severe space-charge limitations and can detect low molecular weight gases, in principle, down to molecular hydrogen.

As a demonstration of the improved sensitivity of the ion trap system, laboratory analyses of water samples collected from Bayboro Harbor during towed deployment of the underwater quadrupole MS system are shown in Figure 7. Although the quadrupole in-situ measurements failed to detect VOCs in the Harbor, laboratory ion trap measurements clearly show the presence of ions $\mathrm{m} / \mathrm{z} 78$ and $m / z$ 91, which are diagnostic of benzene and toluene. At the end of each trace a peak corresponding to injection of $1 \mathrm{ppb}$ standards of these two compounds is shown for 
comparison. Peak intensities for the collected water samples thus correspond to concentrations well below $1 \mathrm{ppb}$ in each case. These results are consistent with those obtained using the in situ quadrupole system, which has a detection limit in the 1-5 ppb range.

The first underwater deployment of the ion trap system was on the OEX AUV in Tampa Bay, using procedures similar to those developed in previous quadrupole system AUV deployments. In situ membrane-introduction ion trap data were collected on four separate deployments, each lasting from 0.5 to 2 hours. One of the AUV/MS deployments involved a pointsource release of 18 liters of dimethylsulfide (DMS) in Tampa Bay. The AUV was programmed to traverse a "lawnmower" pattern across the expected DMS plume. The fluid-switching valve was set to continuously direct bay water to the membrane introduction interface (no deionized water was used in these measurements). There was at least one peak in the data sets for $m / z 62$ and $m / z$ 91, and possible minor peaks. These peaks appear at different times in the deployment, which is more consistent with real chemical concentration changes rather than instrumental fluctuations. We are presently in the process of correlating these data sets with AUV position and modeled distribution of DMS.

To our knowledge, these measurements constitute the first underwater chemical observations obtained using an ion trap mass spectrometry system. Future deployments of both the quadrupole and ion trap MS systems are planned on autonomous and remotely controlled mobile platforms, as well as towed and moored platforms. These deployments will take place in a variety of aqueous systems, including fresh water, saltwater, and waste-water treatment facilities.

\section{Conclusion}

The feasibility of performing in-water mass spectrometry on mobile platforms has been demonstrated using two prototype membrane-introduction MS systems. The quadrupole mass filter and ion trap MS membrane-introduction systems provide $\mathrm{ppb}$ and sub-ppb detection limits, respectively, for most VOCs and dissolved gases. Although analysis cycle times are typically on the order of 5-15 minutes for the present underwater MS systems, real-time in situ analysis provides mass spectrometric information at a rate that is orders of magnitude faster than conventional sampling and laboratory analysis. Our modular approach to underwater mass spectrometer development allows versatile system reconfiguration and future expansion of analytical capabilities. Evolving activities include (1) adaptation of the membrane introduction system and pressure housings for operation at extreme depths, (2) incorporation of a gas chromatograph for detection and quantification of semi-volatile compounds, (3) use of an electrospray source for ion trap MS analysis of non-volatile species, and (4) extension of the mass range of underwater electrospray analyses through time-of-flight mass spectrometry.
Miniaturization of system components is a continuing goal in the evolution of underwater mass spectrometers. We ultimately envision that unmanned underwater vehicle technology will evolve to produce networks of autonomous sensor systems capable of chemically mapping the earth's oceans and, using adaptive sampling strategies, tracing chemicals to their sources. Small underwater MS systems might also one day prove useful in identifying chemical clues in the search for extraterrestrial life, for example in the oceans beneath the ice of Jupiter's moon Europa.

\section{Acknowledgments}

Financial support was provided by the Office of Naval Research to the University of South Florida through a grant N00014-98-1-0154. The authors would also like to acknowledge the contributions and technical assistance of COT members Scott Samson, Gottfried Kibelka, Jim Patten, John Diaz, Randy Russell, Eric Steimle, Charlie Jones and Joe Kolesar. Assistance in AUV deployment of the mass spectrometer from FAU personnel and Tom Peacock of USF is also gratefully acknowledged. The helpful comments of R. G. Cooks are also gratefully acknowledged.

\section{References}

1. Grasshoff, K; Ehrhardt, M; Kremling, E.; Ed. Methods of Seawater Analysis, 2nd ed.; Verlag Chemie $\mathrm{GmbH}$, Weinheim, Germany, 1983.

2. Johnson, K. S.; Coale, K. H.; Jannasch, H. W. Anal. Chem. 1992, 64(22), 1065-1075.

3. Barschick, C. M.; Duckworth, D. C.; Smith, D. H; Ed. Inorganic Mass Spectrometry: Fundamentals and Applications Marcel Dekker, Inc., 2000

4. Norhoff, E.; Kirpekar, F.; Roepstorff, P. Mass Spectrom. Rev. 1996, 15, 67-138.

5. Limbach, P. A. Mass Spectrom. Rev. 1996, 15, 297-336.

6. Murray, K. K. J. Mass Spectrom. 1996, 31, 1203-1215.

7. Lopez-Avila, V.; Hill, H. H. Anal. Chem. 1997, 69(12), 289 R.

8. Badman, E. R.; Cooks, R. G. J. Mass Spectrom. 2000, 35, 659-671.

9. Johnson, R. C.; Cooks, R. G.; Allen, T. M.; Cisper, M. E.; Hemberger, P. H. Mass Spectrom. Rev., 2000, 19(1), 1-37.

10. Bauer, S.; Solyom, D. Anal. Chem. 1994, 66, 4422-4431.

11. Soni, M.; Bauer, S.; Amy, J. W.; Wong, P.; Cooks, R. G. Anal. Chem., 1995, 67(8), 1409-1412.

12. Short, R. T.; Fries, D. P.; Toler, S. K.; Lembke, C. E.; Byrne, R. H. Meas. Sci. Tech. 1999, 10, 1195-1201.

13. Fries, D. P.; Short, R. T.; Byrne, R. H.; Lembke, C. E.; Kerr, M. L. Proceedings of the 48th ASMS Confererence on Mass Spectrometry and Allied Topics, Long Beach, CA, 2000.

14. Camilli, R. Oceans 2000 IEEE/MTS Conference, Student Poster Session, Providence Rhode Island, 2000; Hemond, H; Personal Communication.

15. Louris, J. N.; Cooks, R. G.; Syka, J. E. P.; Kelley, P. E.; Stafford, G. C.; Todd, J. F. J. Anal. Chem., 1987, 59, 1677-1685.

16. Louris, J. N.; Brodbelt-Lustig, J. S.; Cooks, R. G.; Glish, G. L.; Van Berkel, G. J.; McLuckey, S. A. Ion Processes, Int. J. Mass Spectrom. 1990, 96, 117-137.

17. Smith, S. M.; Dunn, S. E. The Ocean Explorer AUV: A Modular Platform for Coastal Sensor Deployment, Autonomous Vehicles in Mine Countermeasures Symposium, 1995, Naval Postgraduate School.

18. Dunn, S. E.; Smith, S. M.; Hopkins, T. Design of Autonomous Underwater Vehicles for Coastal Oceanography Underwater Robotic Vehicles: Design and Control, Yuh, J. Ed., TSI Press, 1994; Chap pp 299-326. 